\title{
Nivel de satisfacción y factores que inciden en la elección del servicio de transporte entre estudiantes de la Universidad de Cartagena, sede Piedra de Bolívar ${ }^{1}$
}

\section{acceso $\mathbf{O}$ abierto}

Para citaciones: Caballero, J., Vergara, N., Coley, J., Pérez, P., Asprilla, I., Maza, F. (2020). Nivel de satisfacción y factores que inciden en la elección del servicio de transporte entre estudiantes de la Universidad de Cartagena, sede Piedra de Bolívar. Revista de jóvenes investigadores Ad Valorem, 3(2), 84-99

Editor: Bernardo Romero Torres. Universidad de Cartagena-Colombia.

Tipología IBN Publindex:

Artículo de investigación científica y tecnológica

Copyright: (C) 2020. Caballero, J., Vergara, N., Coley, J., Pérez, P., Asprilla, I., Maza, F. Este es un artículo de acceso abierto, distribuido bajo los términos de la licencia Creative Commons AtribuciónNoComercial-SinDerivados 4.0 la cual permite el uso sin restricciones, distribución y reproducción en cualquier medio, siempre y cuando que el original, el autor y la fuente sean acreditados.

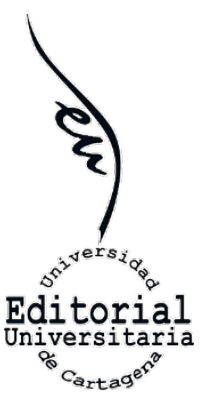

\author{
Juan Camilo Caballero Rueda ${ }^{2}$, Nathalia Andrea Vergara Harris ${ }^{3}$, Jonathan David Coley \\ Vives $^{4}$, Paula Alejandra Pérez Almario ${ }^{5}$, Ivan Camilo Asprilla Riascos ${ }^{6}$, Francisco Javier \\ Maza Ávila ${ }^{7}$
}

Universidad de Cartagena - Colombia

\section{RESUMEN}

En este artículo se analizan los hábitos de movilidad de los estudiantes de los programas de pregrado presenciales de la Universidad de Cartagena, sede Piedra de Bolívar, para determinar los factores que inciden la hora de elegir un medio de transporte para asistir a la institución, así como los niveles de satisfacción que tienen con relación al medio de transporte elegido. Para ello, se aplicó un cuestionario, mediante la técnica encuesta, a una muestra piloto de 100 estudiantes de los programas presenciales de las facultades de Ingeniería y Ciencias Económicas, que funcionan en la sede Piedra Bolívar de la Institución. Los resultados arrojaron altos índices de uso del Sistema Integrado de Transporte Masivo -SITM- Transcaribe y de mototaxi para la movilidad de los estudiantes. Vale señalar que quienes utilizan Transcaribe, lo hacen por la sensación de seguridad y los factores tangibles. Por su parte, los usuarios del servicio de mototaxi señalan que valoran de este medio la velocidad y la corta duración de sus recorridos. No obstante, se identificaron situaciones problemáticas que aquejan a los estudiantes, de las cuales resalta la inconformidad con los tiempos de espera inherentes al uso de los medios de transporte.

Palabras clave: Cartagena de Indias; Elección; Niveles de Satisfacción; Percepciones; Servicio de Transporte.

\footnotetext{
${ }^{1}$ Este documento participó, en la modalidad Proyectos con Resultados, en las XI Jornadas de Investigación de la Facultad de Ciencias Económicas de la Universidad de Cartagena, año 2020

2 Estudiante del programa de Administración Industrial de la Universidad de Cartagena, Colombia. E-mail: jcaballeror@unicartagena.edu.co

3 Estudiante del programa de Administración Industrial de la Universidad de Cartagena, Colombia. E-mail: nvergarah@unicartagena.edu.co

4 Estudiante del programa de Administración Industrial de la Universidad de Cartagena, Colombia. E-mail: jcoleyv@unicartagena.edu.co

5 Estudiante del programa de Administración Industrial de la Universidad de Cartagena, Colombia. E-mail: ppereza1@unicartagena.edu.co

${ }^{6}$ Estudiante del programa de Administración Industrial de la Universidad de Cartagena, Colombia. E-mail: iasprillar@unicartagena.edu.co

${ }^{7}$ Doctor en Ciencias Sociales y Jurídicas, Universidad de Cádiz, España. Docente investigador adscrito al programa de Administración Industrial de la Facultad de Ciencias Económicas y al Instituto Internacional de Estudios del Caribe, Universidad de Cartagena, Colombia. Director del Grupo de Investigación en Estudios para el Desarrollo Regional GIDER-. E-mail: fmazaa@unicartagena.edu.co
} 


\title{
Level of satisfaction and factors influencing the choice of transport service among students at the University of Cartagena, Piedra de Bolívar Headquarters
}

\begin{abstract}
This article analyzes the mobility habits of students in undergraduate programs at the University of Cartagena, Piedra de Bolivar headquarters, to determine the factors that influence the choice of a means of transport to attend the institution, as well as the levels of satisfaction they have with the means of transport chosen. To this end, a questionnaire was applied through the survey technique to a pilot sample of 100 students in the faculty programs of Engineering and Economics Sciences, which operate in the headquarters of the Piedra Bolivar institution. The results showed high rates of use of the Integrated System of Massive Transport -SITM- Transcaribe and of motorcycle informal taxi services for the mobility of the students. It is worth noting that those who use Transcaribe do so because of the feeling of safety and tangible factors. On the other hand, users of the motorcycle informal taxi services indicate that they value the speed and short duration of their routes. Nevertheless, problematic situations were identified that afflict students, of which the non-conformity with the waiting times inherent to the use of the means of transport stands out.
\end{abstract}

Key words: Cartagena de Indias; Choice; Perceptions; Satisfaction levels; Transport Service.

\section{INTRODUCCIÓN}

El crecimiento del parque automotor en las ciudades y la expansión de ellas exige que, a diario, la población se enfrenten decisiones relacionadas a la práctica de movilidad. Se trata de la elección que, a la postre, va a incidir directamente en la movilización hacia y desde distintos lugares; es por ello que esta necesidad de desplazarse crece, y con ella, factores que juegan en contra -y a favor- de la decisión tomada (Maza et al., 2019). Los medios de transporte -tanto formales, como informales-, se ven afectados por múltiples variables que incluyen la seguridad, calidad y percepción en lo concerniente a movilidad (Maza et al., 2019b). Por su parte, son ellas -las variables- quienes determinan la elección del servicio correspondiente y las que paulatinamente forjan la cotidianidad (Gakenheimer, 1998). Es pertinente señalar que la exposición al núcleo urbano desde los distintos tipos de transporte va en crecimiento, pues la inequitativa distribución geográfica y la irregular expansión demográfica, se han constituido en factores que ratifican la heterogeneidad del espacio geográfico al que se hace frente (Manrique, Martínez y Ospina, 2007).

La población estudiantil cada día va en aumento; asimismo, las prácticas de movilidad se expanden y conforman una problemática, dado al alto flujo de estudiantes que representan una proporción significativa en materia de movilidad, y particularmente en Cartagena de Indias -contexto en el que se 
enfocará el trabajo investigativo-, donde el 66\% de los estudiantes matriculados en educación superior pertenecen a estudiantes universitarios, y de ellos, el 31\% corresponden a estudiantes de la Universidad de Cartagena, lo cual se constituyen en datos que dan cuenta de la significativa relevancia que tiene como población cuya apremiante necesidad de desplazarse se materializa a diario (CCV, 2017).

Por lo anterior, la realización de la investigación propuesta, toma como punto de partida la población previamente descrita, en aras de exponer los factores que determinan las condiciones de la elección del medio transporte utilizado para concretar sus desplazamientos hacia y desde la Universidad de Cartagena- sede Piedra de Bolívar, y la valoración de los medios, lo cual, al tener como resultados conocimientos aplicables al campo de estudio (movilidad), enriquece la comunidad científica al contribuir con el avance de problemáticas cotidianas y sobretodo recientes, pues basados en ellos, se ponen de manifiesto las circunstancias en las que se ven inmersas los estudiantes, quienes representan una gran proporción poblacional.

El desarrollo del proyecto contribuye al enriquecimiento académico al no sólo formar parte del mismo entorno, sino al tener como protagonistas a estudiantes, actores principales del campo en estudio. Por su parte, se pretende identificar los elementos que inciden en la percepción de satisfacción del medio de transporte elegido, en términos de eficiencia, seguridad y oportunidad, entre otros, en conjunto con los factores que determinan que la elección del medio escogido se constituya en una decisión u obligación.

\section{Metodología}

Este estudio estuvo compuesto por tres etapas principales. La primera consistió en un análisis observacional de las preferencias y tendencias de uso del transporte en los estudiantes. La segunda consistió en la aplicación de un cuestionario con preguntas abiertas y cerradas, dirigido a estudiantes de los programas presenciales de la Universidad de Cartagena sede Piedra de Bolívar, considerando 3 aspectos primordiales de la investigación, como lo son, los tipos de transporte usados, los factores de elección y la satisfacción y percepción relacionada al transporte usado por los estudiantes. Para ello, se asumieron como criterios de inclusión a aquellos estudiantes pertenecientes a la Facultad de Ciencias Económicas o a la Facultad de Ingeniería de la Universidad de Cartagena. Se excluyeron los estudiantes de programas a distancia y aquellos que cursaban primer semestre. Los primeros, dada la modalidad de estudio a la cual pertenecen, lo que no los obliga a una movilidad diaria para asistir a la institución (sólo asisten a la institución los sábados); los segundos, por el corto periodo que llevan expuestos a el ejercicio de movilidad diaria, y por tanto, aún se encuentran en proceso de elección de rutas y formas de traslado desde y hacia la universidad. 
El cálculo de la muestra para una población finita ascendió a 250 estudiantes (Nivel de confianza del 95\%; error del 5\%). De ella se tomó una muestra piloto de 100 estudiantes, bajo un muestreo estratificado, siguiendo como criterio los distintos horarios de los programas presenciales de la sede Piedra de Bolívar diurno, vespertino y nocturno-.

\section{Resultados}

\section{1. $\quad$ Tipo de transporte usado por los estudiantes}

Los resultados obtenidos a partir del cuestionario aplicado a una muestra de 100 estudiantes de la Universidad de Cartagena, sede Piedra de Bolívar, arrojaron que la población se distribuye a lo largo del día por dicha sede con mayor frecuencia en la jornada diurna y vespertina con un 39\% en ambos casos, dejando como minoría a aquella población que reparte su día en más de una jornada, conformando el 7\% de la población (Gráfico 1).

Gráfico 1. Jornada de estudio de los estudiantes de la Universidad de Cartagena, sede

Piedra de Bolívar

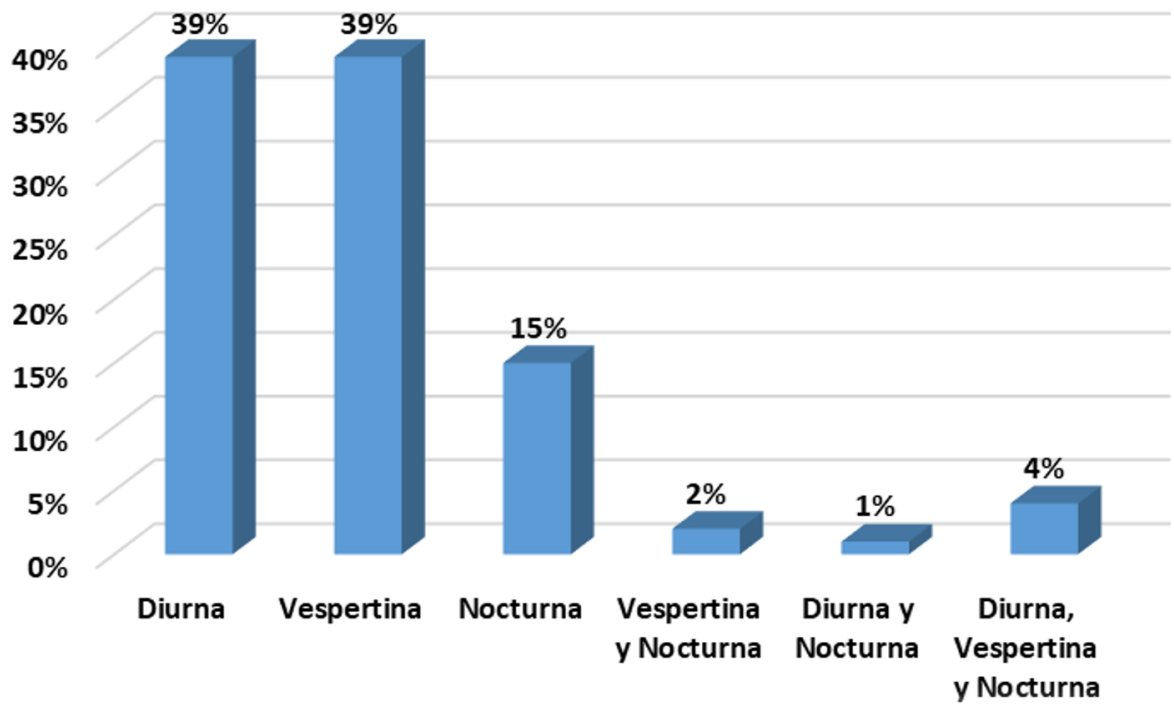

Fuente: elaboración propia, a partir de cuestionarios aplicado a estudiantes

Vale la pena indicar que los encuestados se distribuyen de manera no uniforme por lo largo y ancho las unidades comuneras de gobierno, a partir de este punto denominadas -UCG-, en la ciudad de Cartagena de Indias, concentrándose en la UCG \#8 (15,1\%), a la cual hacen parte barrios como Zaragocilla, Escallón Villa, La Campiña, Los Calamares, entre otros. En conjunto, se encontró que la muestra tomada de estudiantes de la Universidad de Cartagena, sede Piedra de Bolívar, agrupa todos los estratos socioeconómicos desde el 1 hasta el 6, concentrándose el mayor porcentaje en el estrato 2 -45,74\%- (Gráficos 2 y 3). 
Gráfico 2. Residencia de los estudiantes de la Universidad de Cartagena, sede Piedra de Bolívar, por Unidad Comunera de Gobierno -UCG-

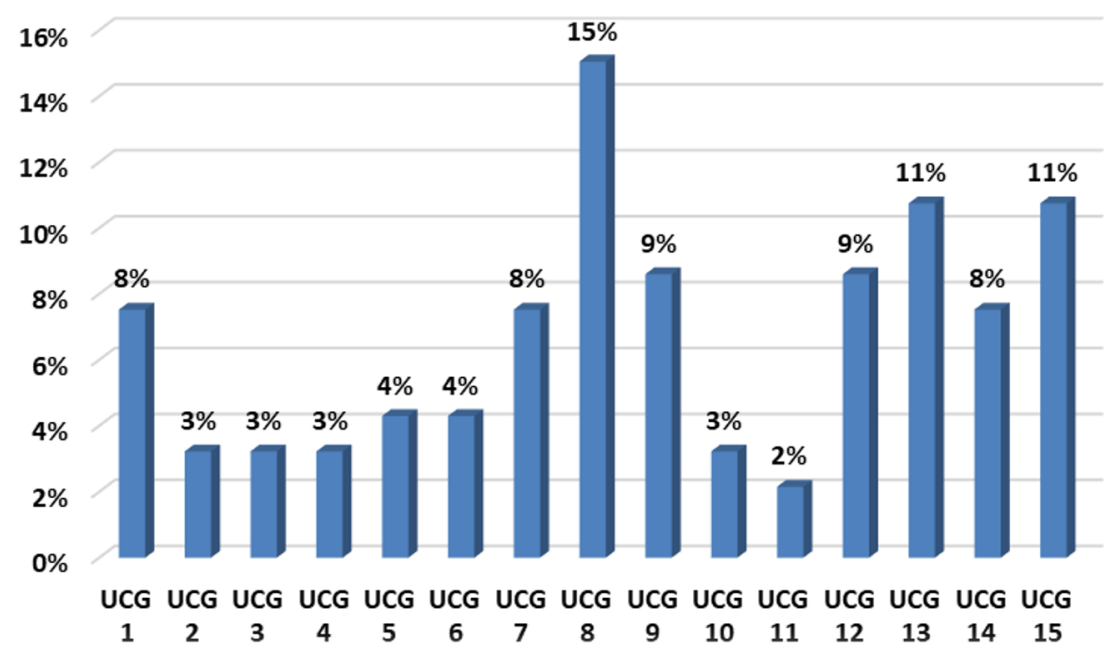

Fuente: Elaboración propia, a partir de cuestionarios aplicado a estudiantes

Gráfico 3. Estrato de los estudiantes de la Universidad de Cartagena, sede Piedra de Bolívar

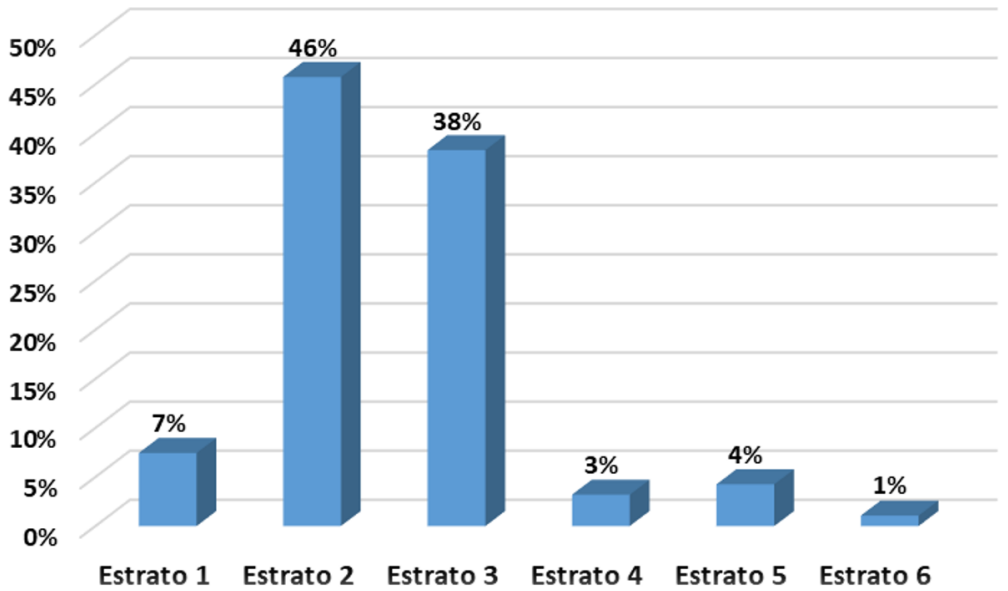

Fuente: Elaboración propia, a partir de cuestionarios aplicado a estudiantes

En materia del servicio de transportes, teniendo en cuenta la zona de residencia de la muestra encuestada, se encontró que la disponibilidad de estos varía en relación a la zona de residencia y el sentido de la ruta -desde o hasta la Universidad de Cartagena-. Se evidencia una clara tendencia en ambos sentidos, mostrando cómo los transportes con mayor disponibilidad los servicios de mototaxi y Transcaribe con un porcentaje de presencia del 89\% y 75\% respectivamente (Gráficos 4 y 5). En relación a las unidades comuneras de gobierno, en Cartagena de Indias -UCG-, se observa que existe relación entre ellas y los transportes disponibles hasta la Universidad de Cartagena (prueba exacta de Fisher, con nivel de significancia del 5\% y p-valor=0,00147). 
Gráfico 4. Transportes disponibles hacia la Universidad de Cartagena, sede Piedra de Bolívar

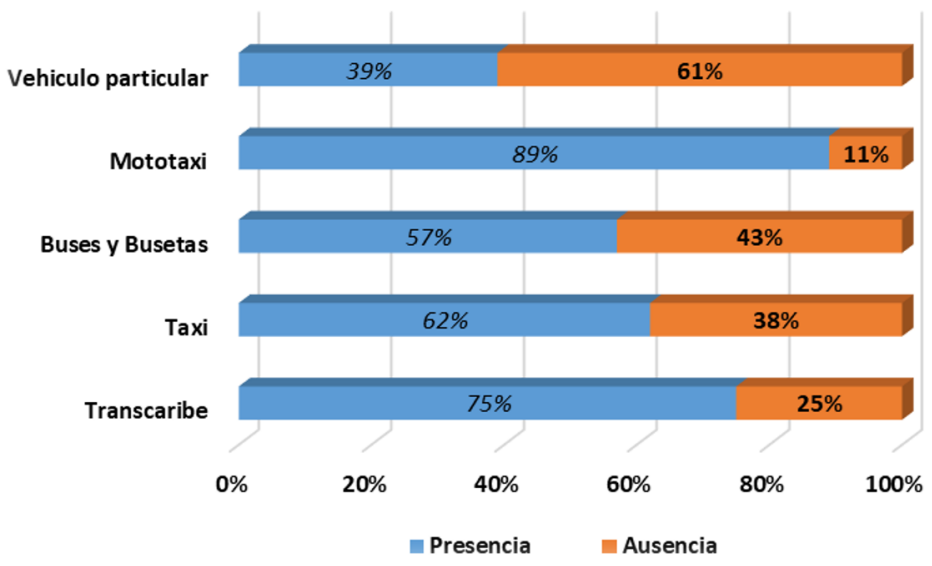

Fuente: Elaboración propia, a partir de cuestionarios aplicado a estudiantes

Gráfico 5. Transportes disponibles desde la Universidad de Cartagena, sede Piedra de Bolívar

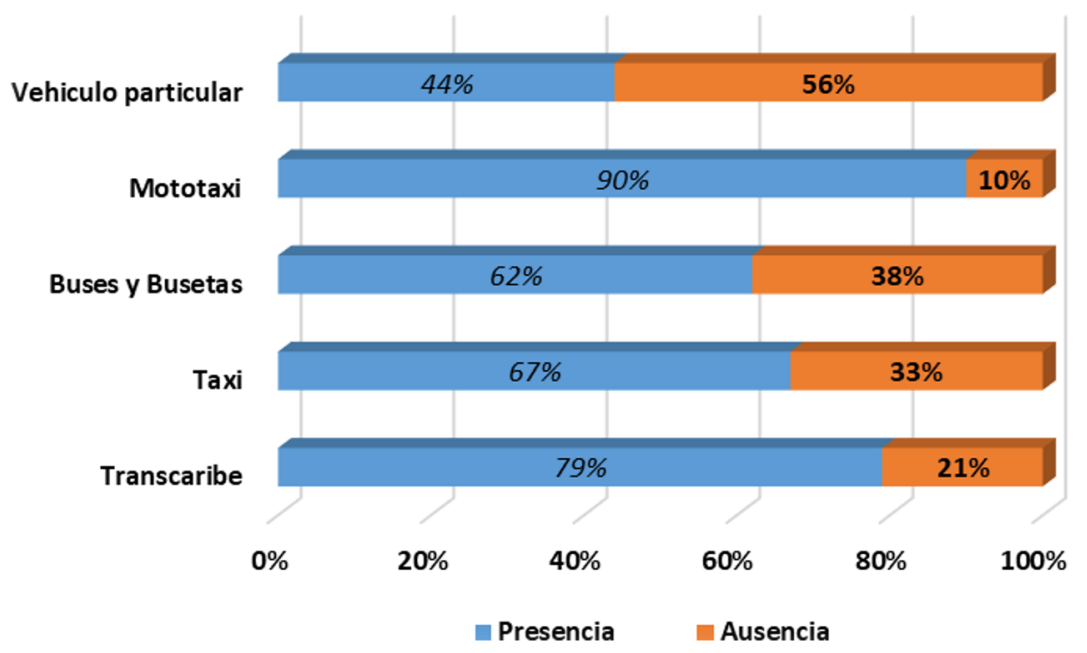

Fuente: Elaboración propia, a partir de cuestionarios aplicado a estudiantes

Una vez señalados los transportes disponibles por cada unidad comunera de gobierno de la ciudad de Cartagena, se hace pertinente caracterizar el número de transportes utilizados para mayor completitud de la investigación en curso. Se pudo observar que la cantidad de transportes utilizados no varía, sin embargo, su proporción sí lo hace. En este sentido, en dirección a la Universidad de Cartagena, la cantidad predominante de servicios de transporte utilizados es de un solo transporte $(55,91 \%)$, seguido por el porcentaje de personas que usan dos transportes (31,18\%); por su parte, en dirección al lugar de residencia desde la Universidad de Cartagena, sede Piedra de Bolívar, el 56,99\% de los estudiantes utiliza un servicio de transporte, seguido de un 29,03\%, quienes utilizan dos medios (Gráficos 6 y 7 ). 
Gráfico 6. Número de transportes utilizados hasta la Universidad de Cartagena, sede Piedra de Bolívar, por Unidades Comuneras (UCG) de Cartagena de Indias

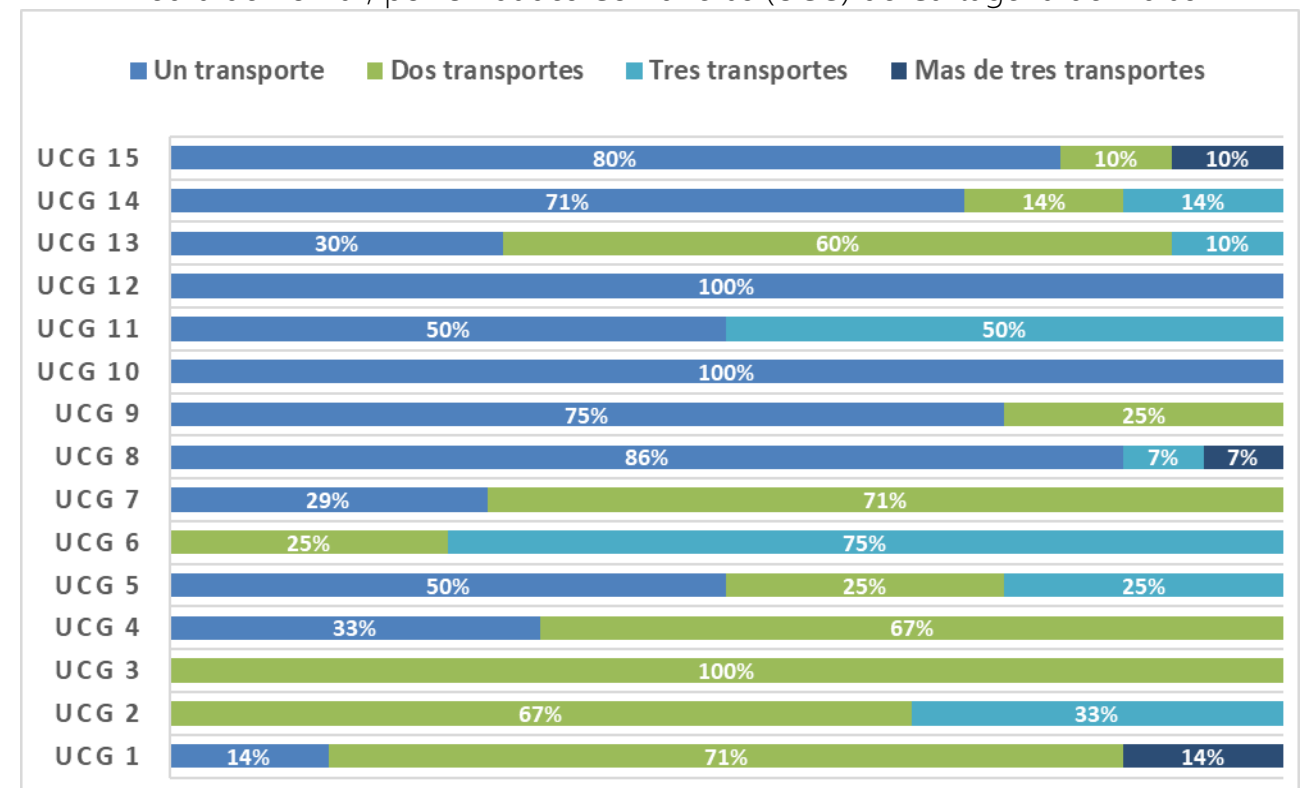

Fuente: Elaboración propia, a partir de cuestionarios aplicado a estudiantes

Gráfico 7. Número de transportes usados desde la Universidad de Cartagena, sede Piedra de Bolívar, por Unidades Comuneras (UCG) de Cartagena de Indias

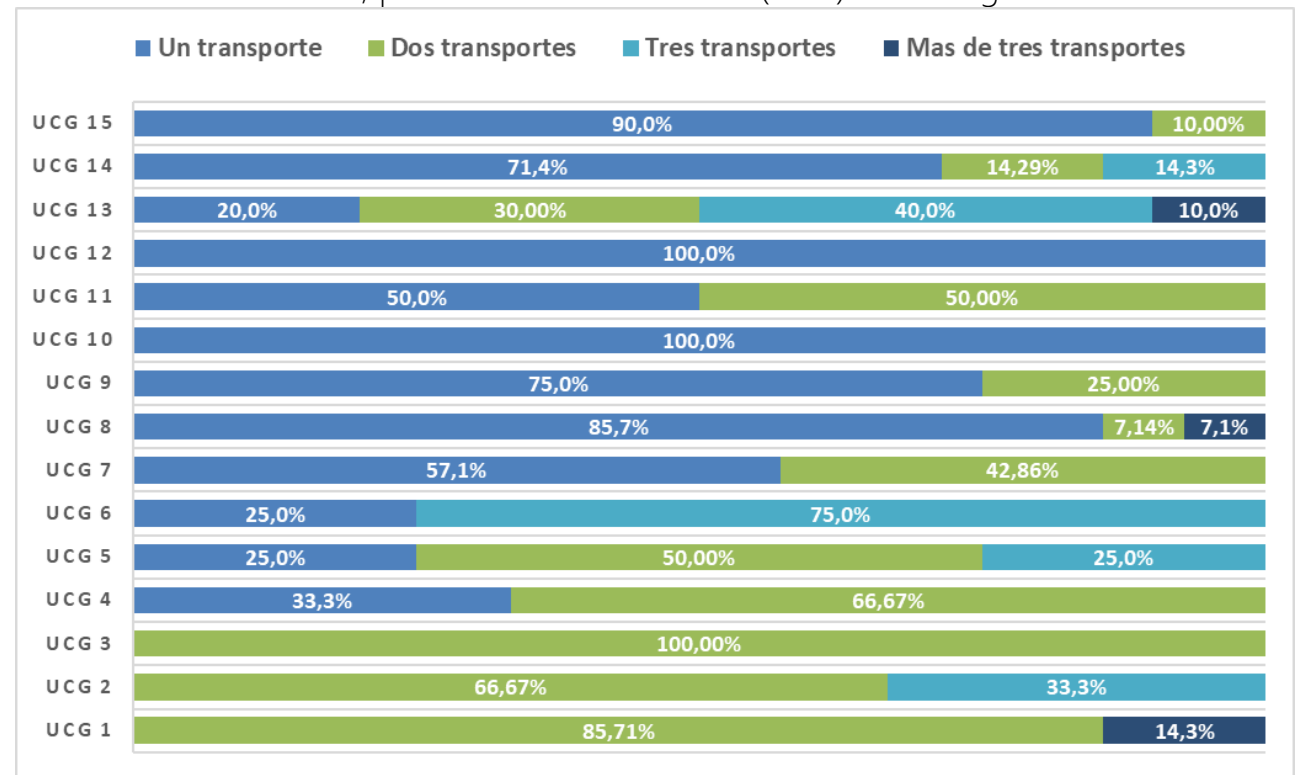

Fuente: Elaboración propia, a partir de cuestionarios aplicado a estudiantes

Teniendo en cuenta las unidades comuneras de gobierno -UCG-, se encontró dependencia estadística entre las mismas y la cantidad de transportes utilizados desde y hasta la Universidad de Cartagena (prueba exacta de Fisher, con nivel de significancia del 5\%), Indicándonos que el número de transportes usados por los estudiantes en ambos sentidos del recorrido depende de la ubicación de su lugar de residencia. 


\subsection{Factores que influyen en la elección del servicio de transporte}

En los resultados obtenidos en la aplicación del cuestionario se puede destacar que, en materia de los ingresos promedio semanales en la muestra estudiada, el $48 \%$ de cuenta con ingresos semanales menores a 25.000 pesos y que además un poco más de la mitad de la muestra -exactamente el 64\%- gasta semanalmente en promedio entre 20.000 y 40.000 pesos, lo que da a conocer el factor costo a pagar. (Gráficos 8 y 9).

Gráfico 8. Ingreso semanal de los estudiantes de la Universidad de Cartagena sede Piedra de Bolívar (en miles de pesos)

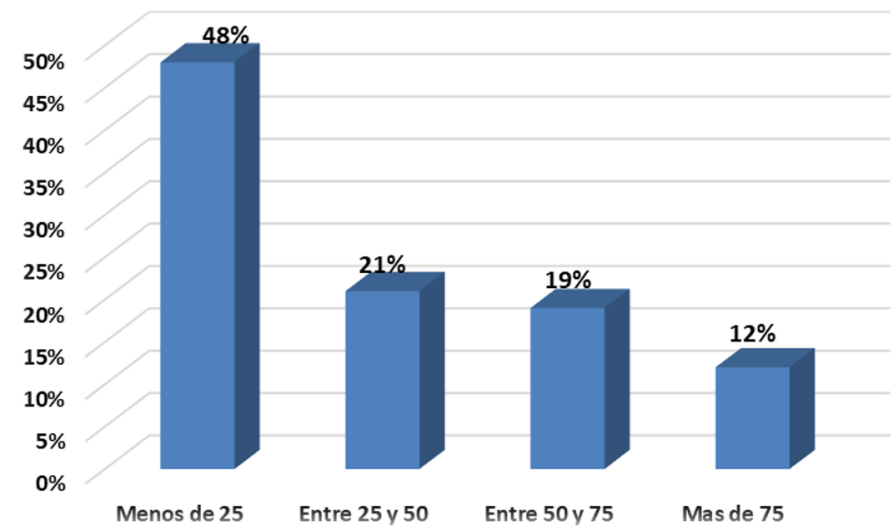

Fuente: Elaboración propia, a partir de cuestionarios aplicado a estudiantes

Gráfico 9. Gasto semanal en transporte por los estudiantes de la Universidad de Cartagena sede Piedra de Bolívar

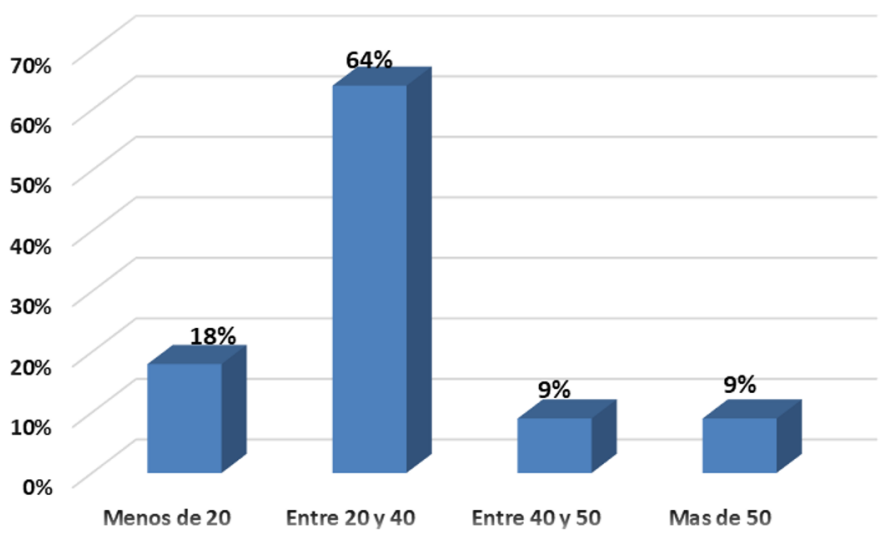

Fuente: Elaboración propia, a partir de cuestionarios aplicado a estudiantes

En relación al ingreso de los estudiantes y el costo a pagar por los estudiantes, no se observó una relación existente entre estos factores y el medio de transporte elegido por la comunidad estudiantil, por lo cual no se pueden señalar como factores determinantes a la hora de la elección del medio de transporte (prueba exacta de Fisher, con nivel de significancia del 5\%). 
Al momento de analizar los periodos de tiempo que los estudiantes empleaban para asistir diariamente a la universidad, se observó que dichos tiempos se distribuían por diferentes magnitudes, asociándose de manera estadística a las UCG de las cuales pertenecen cada uno de los estudiantes, mostrando un variado y parejo espectro en cuanto a demora de transporte se refiere, tomando en cuenta ambos sentidos del ejercicio de movilidad (Gráfico 10).

Gráfico 10. Distribución de tiempo necesario para asistir a la Universidad De Cartagena sede Piedra De Bolívar

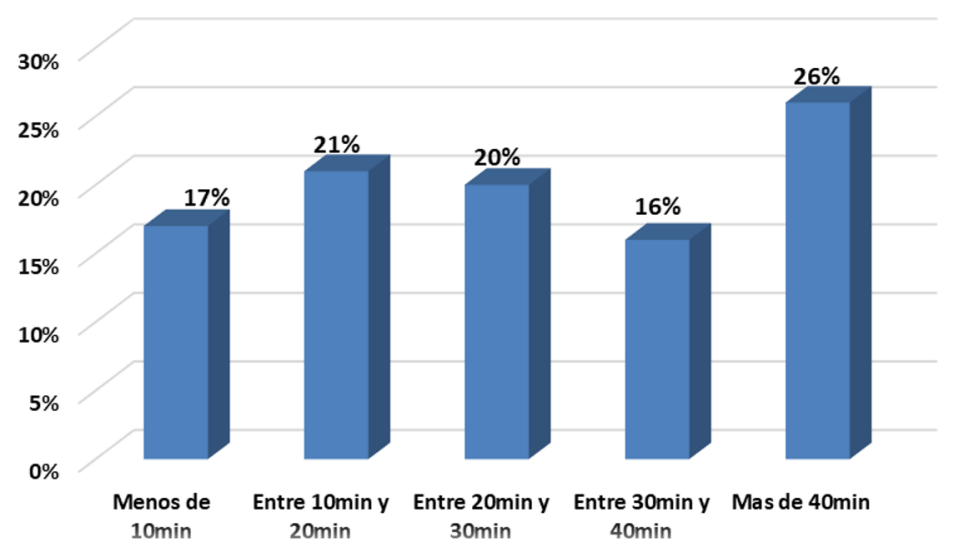

Fuente: Elaboración propia, a partir de cuestionarios aplicado a estudiantes

En relación al factor velocidad, se observó que existe una dependencia con el transporte elegido al momento de dirigirse a sus hogares, pero se tiene evidencia que indique que los estudiantes priorizan la velocidad del transporte al momento de dirigirse a la Universidad De Cartagena Sede Piedra de Bolívar (Prueba exacta de Fisher con nivel de significancia del 5\% p-valor1=0.050, p-valor 2=0.124).

Este estudio permitió establecer una relación estadística entre la importancia que le dan los estudiantes a la velocidad y corta duración de su medio de transporte elegido, y el uso del mototaxi como medio de transporte predilecto (Prueba exacta de Fisher con nivel de significancia del 5\% p-valor1 $=0.041$, p-valor $2=$ 0.050, p-valor $3=0.0351$, p-valor $4=0.03$ ). Por lo cual se puede concluir que la población estudiantil que antepone la velocidad y la corta duración al momento de hacer la elección de su medio de transporte para asistir a la universidad de Cartagena, mostró una clara inclinación por el uso frecuente del mototaxi. También se pudo identificar una relación muy fuerte entre el tiempo promedio de duración del recorrido y la percepción que tienen los estudiantes sobre este mismo (Prueba exacta de Fisher con nivel de significancia del 5\% p-valor1=0.001), mostrando que los estudiantes que se ven obligados a un mayor tiempo de recorrido, tienen una peor percepción sobre este factor como se muestra en el Gráfico 11. 
Gráfico 11. Satisfacción de los estudiantes sobre la duración promedio del recorrido

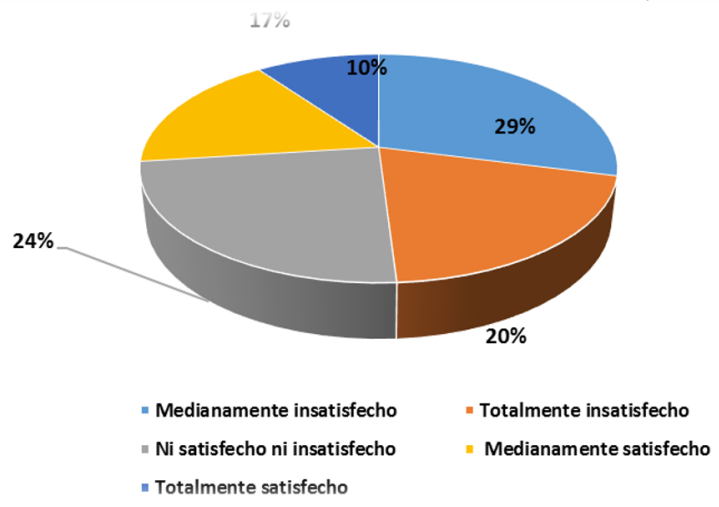

Fuente: Elaboración propia, a partir de cuestionarios aplicado a estudiantes

\subsection{Satisfacción en relación al medio de transporte usados por los estudiantes}

En este apartado se pretende analizar el nivel de satisfacción del medio de transporte utilizado por los estudiantes, considerando criterios tales como la novedad de los equipos que prestan el servicio, el atractivo físico del vehículo y la percepción en torno a las condiciones ambientales de la entidad prestadora del servicio. Se encontraron relaciones significativas entre el uso del SITM y una buena percepción de los todos factores tangibles. Como contraparte, los resultados revelaron que los estudiantes que hacen uso frecuente mototaxis, valoran negativamente la calidad de sus elementos tangibles; por último, cabe resaltar una relación negativa entre el uso de los buses y la percepción relacionada con la condición física del medio empleado, así como de las condiciones climáticas ofrecidas.

En cuanto a la capacidad de respuesta, los resultados arrojaron satisfacción general en la mayoría de los aspectos evaluados en la encuesta, a excepción de los tiempos de espera, el cual presentó una gran tasa de insatisfacción, cercana al 50\% (Gráfica 12)

Gráfico 12. Valoración de los tiempos de espera y/o transbordo en los servicios de transporte
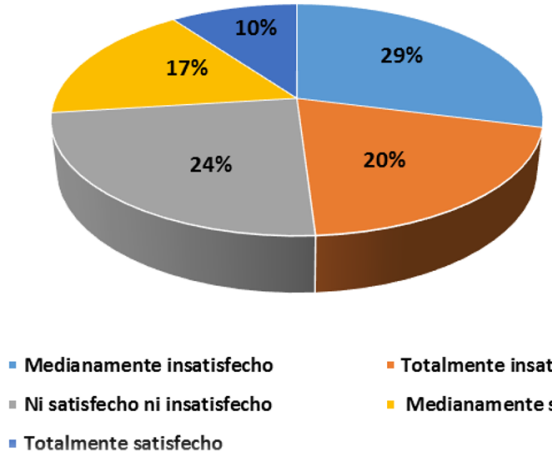

- Totalmente insatisfecho

- Medianamente satisfecho

Fuente: Elaboración propia, a partir de cuestionarios aplicado a estudiantes 
La empatía, por su parte, se evaluó tomando como criterios la Percepción de la accesibilidad del servicio de transporte, Percepción de la comunicación ofrecida por la empresa prestadora del servicio de transporte y la Percepción de la comprensión ofrecida al cliente por las empresas prestadoras del servicio de transporte, lo cual mostró resultados favorables, en términos generales (Gráfico 13).

Gráfico 13. Valoración de la empatía en los servicios de transporte

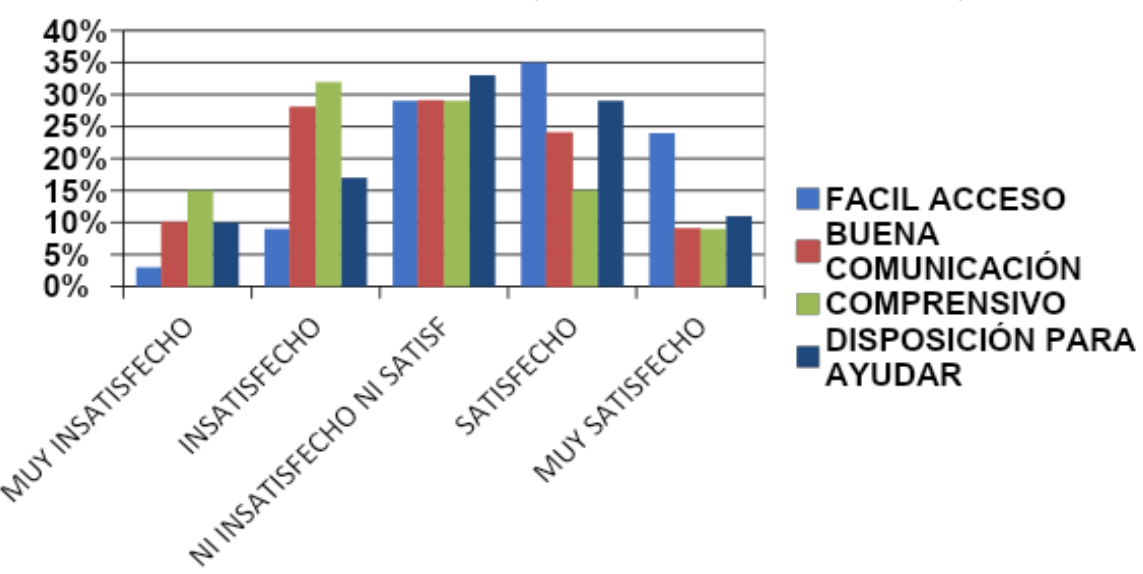

Fuente: Elaboración propia, a partir de cuestionarios aplicado a estudiantes

Por último, se evaluó la seguridad teniendo en cuenta aspectos como la percepción del profesionalismo de los operarios del sistema de transporte elegido, percepción de la cortesía presente en los operarios del sistema de transporte elegido, percepción de la credibilidad en el servicio de transporte que se proporciona y la percepción de la seguridad ofrecida por el sistema de transporte elegido. Los resultados arrojaron indiferencia por parte de la muestra, pues no se mostraron ni satisfechos ni insatisfechos en torno a la seguridad percibida por el servicio de transporte utilizado.

Gráfico 14. Valoración de la seguridad en los servicios de transporte

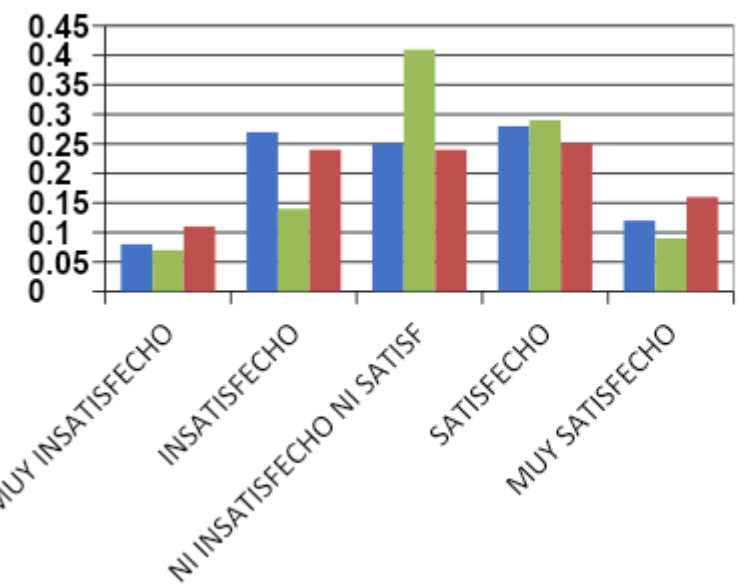

DROFESIONALISMO

Fuente: Elaboración propia, a partir de cuestionarios aplicado a estudiantes 


\section{CONCLUSIONES}

La obligación de movilidad diaria a la cual están sujetos los estudiantes de los programas presenciales de la Universidad de Cartagena, sede Piedra de Bolívar, y las múltiples opciones de transporte que ofrece la vida actual, acusa la toma de decisiones, la cual se ve motivada por diferentes aspectos a tener en cuenta para asegurar la mejor elección en cada caso particular. Con todo esto, se puede concluir que los estudiantes toman como principales criterios de elección de su medio de transporte, la seguridad, la duración del recorrido, la velocidad y los factores tangibles del medio de transporte, todo esto en diferentes medidas, mostrando una inclinación o tendencia de los estudiantes que anteponen la velocidad y un corto tiempo de recorrido al uso de mototaxis como transporte principal para su movilización. A su vez, los estudiantes también basan su decisión en la seguridad de los medios de transporte tipo bus o automóvil. En el caso particular de los estudiantes que le otorgan suma importancia a los factores tangibles propios del vehículo, mostraron una fuerte inclinación por el uso del SITM (Transcaribe S.A.), esto último, debido a la flota de vehículos mejor equipada en comparación al resto de posibles opciones. Lo anterior permite explicar las tendencias de uso inclinadas hacia el SITM (Transcaribe S.A.) y el mototaxi como medios predilectos para este ejercicio de movilidad.

En relación a satisfacción y percepción, los estudiantes se sienten inconformes con los tiempos de espera o transbordo por un medio de transporte, asimismo con la empatía que tienen con el servicio y los avances tecnológicos usados en los mismos. Por último, la percepción de los estudiantes acerca de la seguridad brindada por los medios de transporte es indiferente, puesto que la mayor parte de ellos no se muestran satisfechos o insatisfechos con la seguridad ofrecida.

\section{REFERENCIAS}

Cartagena Cómo vamos (2017). Informe calidad de vida 2017.

Gakemheimer, R. (1998). "Los problemas de la movilidad en el mundo en desarrollo".

Manríquez, Martínez y Ospina (2007). Crecimiento Poblacional y Políticas Públicas.

Maza Avila, F. J., Fals Galezo, M. P., Espinosa Flórez, L. C., Safar Cano, C. F., \& Licona Dáger, D. (2019). Percepciones del riesgo asociado a la práctica del mototaxismo en Cartagena, Colombia. Economía \& Región, 13(2), 57-81. https://doi.org/10.32397/er.vol13.n2.2

Maza Avila, F. J., Blanco Bello, R., \& Fals Galezo, M. P. (2019b). Percepciones sobre los efectos económicos, sociales y ambientales del mototaxismo en municipios del departamento de Bolívar (Colombia). Revista Panorama Económico 27(2), 349-369. https://dialnet.unirioja.es/servlet/articulo?codigo $=7513390$ 


\section{BIBLIOGRAFÍA}

Amézquita, L. Durán, D. \& Fajardo, D. (2016) Matriz origen-destino y eficiencia en modos de transporte urbano: un análisis de la movilidad de Bogotá.

Arroyo Lara, A. \& Arrieta Puello, R. (2015). Efectos del mototaxismo en el transporte público colectivo urbano y la movilidad en la ciudad de Cartagena de Indias, en las rutas: 15A, 21, 26, 30A, 34, 44B, 44D, 46 y 47.

Arrue (2009). "algunas reflexiones sobre la movilidad urbana en Colombia desde la perspectiva del desarrollo humano" recuperado de http://www.scielo.org.co/pdf/papel/v16n2/v16n2a07.pdf

Barrato, L. (1988). ¿Qué es la calidad? Revista escuela de administración de negocios, no. 6. Pág. 12-17.

Berdugo, S. (2012). Medición de la calidad del proceso de matrícula, mediante la percepción del grado de satisfacción de los estudiantes del Programa de Fisioterapia de la Universidad Manuela Beltrán - UMB (Bogotá D.C.) (Tesis de maestría). Escuela de graduados de educación EGE. Bogotá Colombia.

Bruner, Cols (1958). Enfoques teóricos sobre la percepción que tienen las personas. Horizontes pedagógicos, 8(1).

Carrión, Fernando (2001). "Las nuevas tendencias de urbanización en América Latina". En Carrión, Fernando, La ciudad construida: urbanismo en América Latina. FLACSO, Quito, pp. 7-24.

Contreras, A. (2012). Percepción Directa: El enfoque ecológico como alternativa al cognitivismo en la percepción (Tesis de grado). Universidad de Chile, Santiago, Chile.

De Oña (2013), Titulado "Analysis of service quality in public transportation using decision trees".

Duque Oliva, Edison Jair. (2005). Revisión del concepto de calidad del servicio y sus modelos de medición. Revista de ciencias administrativas y sociales, vol. 15, núm. 25. Pág. 64-80.

Franco, Lila (2014). Titulado "Determinación del Índice de Satisfacción de la Movilidad en el Sistema de Transporte de Estudiantes Universitarios en Venezuela".

Gómez, Hilda (2019). Agencia Nacional de Seguridad Vial. Recuperado de: https://wwwrcnradio-com.cdn.ampproject.org/v/s/www.rcnradio.com/colombia/ansv-alertaque-accidentalidad-en-moto-en-colombia-es-muyelevada?amp_js_v=a2\&amp_gsa $=1$ \&amp\&usqp $=$ mq331AQA\#aoh $=156026251546$ 61\&amp_ct=1560262518594\&referrer=https\%3A\%2F\%2Fwww.google.com\&amp_t f=De\%20\%251\%24s\&ampshare=https\%3A\%2F\%2Fwww.rcnradio.com\%2Fcolombi a\%2Fansv-alerta-que-accidentalidad-en-moto-en-colombia-es-muy-elevada 
Gonzáles, Moreno, Velázquez (2011). "Análisis de la movilidad en campus universitarios: caso de estudio universidad de Antioquia".

Heredia (2015). "Modelo de satisfacción de los usuarios de transporte publico tipo bus integrando variables latentes". Recuperado de http://www.bdigital.unal.edu.co/50005/1/1128278231.2015.pdf

Kotler, Anstromg (1985) "Fundamentos de marketing" recuperado de https://www.promonegocios.net/mercadotecnia/precio-definicion-concepto.html

Departamento de Bolívar (Colombia). Revista Panorama Económico, 27(2), 349-369.

Miralles-Guash, Avellaneda, cebollada (2003). Titulado "los condicionantes de la movilidad en un nodo de la ciudad metropolitana: el caso de la Universidad Autónoma de Barcelona".

Observatorio Nacional de Seguridad Vial -ONSV-(2018). "Boletines estadísticos. fallecidos, lesionados y hechos de tránsito en Colombia".

Organización Mundial de la Salud, (1998). "Seguridad y promoción de la seguridad: Aspectos conceptuales y operacionales" recuperado de:

https://www.inspq.qc.ca/es/centro-collaborador-oms-de-quebec-para-la-promocionde-la-seguridad-y-prevencion-de-traumatismos/definicion-del-concepto-deseguridad

Orozco, Ángela (2019). Ministerio de Transporte, recuperado de: https://www.rcnradio.com/colombia/en-colombia-fallecen-15-personas-al-diapor-accidentes-de-transito.

Puy, A. (1994). Percepción social del riesgo: Dimensiones de evaluación y predicción (Doctoral dissertation, Tesis Doctoral. Departamento de Psicología Social. Facultad de Psicología. Universidad Complutense de Madrid. Madrid).

Rafael Danilo Cedeño Millares, Joaquín Carcacés Domínguez (2010). "La calidad en los servicios de transporte".

Revista Dinero (2018) "¿Cuánto cuesta un pasaje de transporte masivo en Colombia?". Recuperado de https://www.dinero.com/pais/articulo/costo-del-transportemasivo-en-colombia/255232

Revista Médica Herediana (1998) "¿Qué es calidad total?". Recuperado de http://www.scielo.org.pe/scielo.php?script=sci arttext\&pid=S1018$\underline{130 \times 1998000100006}$

Revista periodística Las2Orillas (2017), "El transporte público en Cartagena es una soberana porquería". Recuperado de https://www.las2orillas.co/transportepublico-cartagena-una-soberana-porqueria/ 
RD Millares, JC Domínguez - Eumed. Net (2011). "La calidad de los servicios de transporte".

Salazar, J., Montero, M., Muñoz, C., Sánchez, E., Santoro, E. \& Villegas, J. (2012). Percepción Social, En Psicología Social (pp. 77-109). México: Trillas.

Tamayo y Tamayo (1997) "procesos de investigación científica" recuperado de http://tesisdeinvestig.blogspot.com/2011/06/poblacion-y-muestra-tamayo-ytamayo.html

Titheridge, H. (2004) Social exclusion and transport policy.

\section{Apéndice 1: Selección de población y muestra}

Para la selección de nuestra población de estudio se tendrán en cuenta una serie de criterios de inclusión, de los cuales destaca la pertenencia a la Universidad De Cartagena y que a su vez estén inscritos en programas de la facultad de ingeniería y la facultad de ciencias económicas ya que estas dos son las que se encuentran en el campus de Piedra De Bolívar. Se enfocará en los estudiantes de programas de pregrado presenciales, ya que estos son los que evidencian la obligación de movilizarse diariamente hacia la institución, dejando de lado a los estudiantes de nuevo ingreso debido a que estos al estar en periodo de adaptación a la vida universitaria no han creado aun costumbres de movilidad, generando un posible sesgo en la información recogida. Se escogerá una muestra representativa a la que se le aplicará una encuesta por medio del instrumento de cuestionario. Para que dicha muestra sea representativa se hará uso de la siguiente formula:

$$
n=\frac{z^{2} N p q}{(N-1) e^{2}+z^{2} p q}
$$

Donde:

$\mathrm{p}=$ Probabilidad de que un fenómeno ocurra.

$q=$ Probabilidad de que un fenómeno no ocurra

$\mathrm{N}=$ Tamaño de la población

$\mathrm{n}=$ Tamaño de la muestra

z= Correspondiente al nivel de confianza elegido, que en este caso será del 95\%

$\mathrm{e}=$ Error muestral permitido que para este caso será el 5\%

Para fines de nuestro análisis, se establecerá un intervalo de confianza del 95\%, un error muestral permitido del e $=5 \%$. En este trabajo de investigación, a los valores parámetros $p$ y $q$ se asignarán los valores $p=0.5$ y $q=0.5$. Con un tamaño de población de 4060 estudiantes que cumplen con los criterios de inclusión y exclusión definidos de antemano (SNIES, fecha de corte: 16 de octubre de 2019), nos da un tamaño de muestra $n=254$ estudiantes, para cuestiones de practicidad decidimos tomar una muestra piloto de 100 estudiantes. 


\section{Apéndice 2: Prueba exacta de Fischer}

La prueba de Fisher es el test exacto utilizado cuando se quiere estudiar si existe asociación entre dos variables cualitativas, es decir, si las proporciones de una variable son diferentes dependiendo del valor que adquiera la otra variable. En la gran mayoría de casos, el test de Fisher se aplica para comparar dos variables categóricas con dos niveles cada una (tabla 2×2). Es posible utilizarlo con tablas 2xK niveles, pero los requerimientos de cálculo son altos.

El test de Fisher es más preciso que sus equivalentes aproximados (test chi-square de independencia o G-test de independencia) cuando el número de eventos esperado por nivel es pequeño. Se recomienda utilizarlo siempre que sea posible (tiempo de computación) aunque para observaciones totales >1000 los resultados de los test aproximados son muy parecidos.

El test exacto de Fisher se basa en la distribución hipergeométrica, que permite calcular la probabilidad exacta de obtener una determinada distribución de eventos dentro de una tabla.

$$
p=\frac{\left(\begin{array}{c}
a+b \\
a
\end{array}\right)\left(\begin{array}{c}
c+d \\
c
\end{array}\right)}{\left(\begin{array}{c}
n \\
a+c
\end{array}\right)}=\frac{(a+b) !(c+d) !(a+c) !(b+d) !}{a ! b ! c ! d ! n !}
$$

El test de Fisher calcula las probabilidades de todas las posibles tablas y suma las de aquellas tablas que tengan probabilidades menores o iguales que la tabla observada, generando así el p-valor de dos colas. 